\title{
Dispersion-Free Component of Non-Covalent Interaction via Mutual Polarization of
} Fragments' Densities

\author{
Marcin Modrzejewski, ${ }^{1, a)}$ Łukasz Rajchel, ${ }^{2, b)}$ Małgorzata M. Szczęśniak, ${ }^{3}$ and Grzegorz \\ Chałasiński $^{1}$ \\ 1) Faculty of Chemistry, University of Warsaw, 02-093 Warsaw, Pasteura 1, \\ Poland \\ 2) Interdisciplinary Centre for Mathematical and Computational Modelling, \\ University of Warsaw, 02-093 Warsaw, Pawińskiego 5a, \\ Poland \\ 3) Department of Chemistry, Oakland University, Rochester, Michigan 48309-4477, \\ USA
}

Comprehensive tests within a diverse set of non-covalently bonded systems are carried out to assess the performance of the recently-developed dispersion-free approach in the framework of density functional theory [€. Rajchel, P. Żuchowski, M. Szczęśniak, and G. Chałasiński, Phys. Rev. Lett., 104, 163001 (2010)]. A numerical algorithm which cures the convergence problems of the previous implementation is presented.

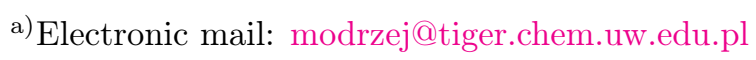

b) Electronic mail: L.Rajchel@icm.edu.pl
} 


\section{INTRODUCTION}

The density functional theory (DFT) has emerged as a powerful and efficient methodology for studying chemical systems since Hohenberg and Kohn ${ }^{1}$ laid out the milestone founding theorems of the theory and came up with the ingenious idea on how to put them to work, back in the 1960s. ${ }^{2}$ The plethora of approximations to the exchange-correlation (xc) functional, the crucial ingredient of the DFT, has been proposed and thoroughly benchmarked (as a recent example, see Ref. 3 and the Refs. therein).

On the one hand, the density functional approximations perform satisfactorily for the determination of geometries, energetics, and static properties of the chemical systems, as well as the interaction energies of hydrogen bonds and electrostatically-bound complexes. ${ }^{4,5}$ On the other hand, the existing xc functionals fail spectacularly to describe the dispersion interactions. ${ }^{6,7}$ The local (in case of local spin-density approximations), or semi-local (for generalized gradient approximations) character of current xc functionals makes them unable to describe the long-range intermonomer correlation effects manifesting themselves as the dispersion interaction. ${ }^{8,9}$ Up to now several approximate non-local functionals seamlessly incorporating dispersion forces have been proposed. ${ }^{10-12}$ Other approaches for solving the dispersion problem within DFT are generally attempts to model the dispersion energy separately. The most successful examples are dispersion-corrected atom-centered potentials, ${ }^{13}$ DFT+dispersion method, ${ }^{14,15}$ exchange hole dipole model, ${ }^{16}$ and DFT-based symmetry adapted perturbation theory (SAPT). ${ }^{17,18}$ See Ref. 19 for a recent comprehensive review of the DFT-based dispersion approaches.

For DFT-based dispersion models to be successful, the proper description of the dispersionfree interaction energy is necessary. In the above-mentioned approaches (with the exception of SAPT) dispersion-free energy is obtained in a supermolecular fashion. Such an energy, however, already includes some fraction of correlation effects which can become doubly counted upon combining with dispersion correction. Moreover, the exchange contribution must be compatible with dispersion correction in the regions important for the interaction energy. ${ }^{20}$ These issues have motivated a few groups to design the DFT method that would rigorously exclude the dispersion interaction. The examples are:

- The dispersionless functional of Pernal et al. ${ }^{21}$ The xc functional is constructed through the fitting to the set of interaction energies from which the dispersion energy 
is subtracted.

- The Pauli blockade (PB) method described in Refs. 22,23. The aims of the method are

- to include the intramonomer correlation,

- to rigorously exclude the intermonomer correlation.

The former goal is accomplished by the KS description of the interacting monomers while the latter is achieved by the use of the HF exchange between the monomers. Consequently, the PB energy is dispersion-free.

- A long-range correction scheme for the exchange functional ${ }^{24-28}$ which was proven to correct the inaccuracies of local exchange potentials in regions of large reduced density gradients, important for intermolecular interactions. ${ }^{20}$ Such calculations lead to energies which can be supplied with a proper dispersion contribution, as was first shown by Kamiya, Tsuneda, and Hirao ${ }^{20}$ (see also Ref. 29).

Below we will focus entirely on the PB approach. Our previous works on the subject did not include any calculations performed within a systematic set of noncovalently bound systems. For this reason, no definite conclusions could be made to date on the general performance of the PB method. In this work, we perform calculations for the well-established test suite of noncovalently bound systems, ${ }^{30,31}$ which will help identify systems for which the PB method performs satisfactorily, and to find the systems for which it should not be applied. Additionally, an efficient algorithm for converging main equation of the PB method is presented. The new algorithm corrects divergent and oscillatory behavior of the previously used penalty function method. ${ }^{32}$

\section{PAULI BLOCKADE FORMALISM}

In what follows, $i(a)$ denotes occupied (virtual) molecular orbital (MO). Greek letters, $\mu$, $\nu$, etc. represent indices of orthogonalized atomic orbitals (OAO) unless indicated otherwise. All matrices are written in the OAO basis, except in cases where the atomic orbital (AO) label is present. 
Consider a system of two weakly interacting molecules, $A$ and $B$, referred to as monomers later on. Density matrices describing the electronic density localized at the monomers will be denoted as $\mathbb{D}_{\zeta}, \zeta=A, B$. These can be used to define the monomer $\mathbb{F}_{\zeta}$ matrix, which differs from the isolated monomer Kohn-Sham matrix by the presence of nuclear attraction operator of the other interacting molecule:

$$
\mathbb{F}_{\zeta}=\mathbb{F}\left(\mathbb{D}_{\zeta}\right)=\mathbb{T}+\mathbb{U}_{A}^{\mathrm{ne}}+\mathbb{U}_{B}^{\mathrm{ne}}+\mathbb{J}\left(\mathbb{D}_{\zeta}\right)+\mathbb{V}^{\mathrm{xc}}\left(\mathbb{D}_{\zeta}\right),
$$

where $\mathbb{T}$ is the matrix of the kinetic energy operator, $\mathbb{U}_{A}^{\text {ne }}$ and $\mathbb{U}_{B}^{\text {ne }}$ are matrices of the nucleielectron attraction operator of monomer $A$ and $B$, respectively, $\mathbb{J}$ is the matrix of the Hartree potential, and finally, $\mathbb{V}^{\mathrm{xc}}$ represents the matrix of the exchange-correlation potential. Using Eq. (1) we set up a system of equations for interacting monomers: ${ }^{22,23}$

$$
\left\{\begin{array}{l}
\left(\mathbb{F}_{A}+\Delta \mathbb{V}_{A}^{\mathrm{xc}}+\mathbb{J}_{B}\right) \mathbb{C}_{i}=e_{i} \mathbb{C}_{i}, \quad i \in A \\
\left(\mathbb{F}_{B}+\Delta \mathbb{V}_{B}^{\mathrm{xc}}+\mathbb{J}_{A}\right) \mathbb{C}_{j}=e_{j} \mathbb{C}_{j}, \quad j \in B
\end{array},\right.
$$

where $\mathbb{C}_{i}$ is a $i$-th column of the MO coefficients matrix of monomer $\mathrm{A}, \mathbb{C}_{\mathbb{A}}$, and similarly for $\mathbb{C}_{j}$. Eq. (2) is equivalent to a single Kohn-Sham equation for the dimer, provided that the occupied orbitals are orthogonal:

$$
\mathbb{C}_{i}^{\dagger} \mathbb{C}_{i^{\prime}}=\delta_{i i^{\prime}}, \quad i, i^{\prime} \in A B .
$$

In the $A$ monomer part of Eq. (2), matrix $\mathbb{J}_{B}$ describes direct Coulomb interaction with electrons localized at monomer $B$. Its matrix element in OAO basis is computed as

$$
J_{B, \mu \nu}=2 \sum_{\tau v} S_{\mu \tau}^{-1 / 2} S_{\nu v}^{-1 / 2} \sum_{\rho \sigma}(\tau v \mid \rho \sigma) D_{B, \sigma \rho}^{\mathrm{AO}},
$$

where $(\tau v \mid \rho \sigma)$ denotes a two-electron integral in Mulliken notation and $S_{\mu \tau}^{-1 / 2}$ is an element of inverse square root of the $\mathrm{AO}$ overlap matrix. $\Delta \mathbb{V}_{A}^{\mathrm{xc}}$ is the matrix of the non-additive exchange-correlation potential:

$$
\Delta \mathbb{V}_{A}^{\mathrm{xc}}=\mathbb{V}^{\mathrm{xc}}\left(\mathbb{D}_{A B}\right)-\mathbb{V}^{\mathrm{xc}}\left(\mathbb{D}_{A}\right)
$$

with the density matrix of dimer $A B$ being

$$
\mathbb{D}_{A B}=\mathbb{D}_{A}+\mathbb{D}_{B}=\mathbb{C}_{\mathrm{occ}, A} \mathbb{C}_{\mathrm{occ}, A}^{\dagger}+\mathbb{C}_{\mathrm{occ}, B} \mathbb{C}_{\mathrm{occ}, B}^{\dagger} .
$$


By $\mathbb{C}_{\text {occ }}$ we denote the matrix of $\mathrm{MO}$ coefficients expressed in OAO basis. The orthogonality of orbitals that belong to different monomers implies idempotency of supermolecule density matrix:

$$
\mathbb{D}_{A B}^{2}=\mathbb{D}_{A B},
$$

as should be for the solution of the Kohn-Sham equation. We emphasize that Eq. (2) is equivalent to a single Kohn-Sham equation for the $A B$ supermolecule. However, the separation into monomer equations is a good starting point for introducing approximations in the context of intermolecular interactions. This work relies on dispersion-free approximation, ${ }^{22}$ in which interaction of monomers is treated at the HF exchange-only level, as the exchangecorrelation non-additivity is approximated by the HF exchange matrix:

$$
\Delta \mathbb{V}_{A}^{\mathrm{xc}} \approx \mathbb{K}_{B}
$$

where

$$
K_{B, \mu \nu}=-\sum_{\tau v} S_{\mu \tau}^{-1 / 2} S_{\nu v}^{-1 / 2} \sum_{\rho \sigma}(\tau \sigma \mid \rho v) D_{B, \sigma \rho}^{\mathrm{AO}} .
$$

The dispersion-free energy of the $A B$ system that corresponds to the approximation of Eq. (8) can be expressed as

$$
\mathcal{E}_{A B}^{\mathrm{PB}}=E_{A B}^{\mathrm{HF}}\left(\mathbb{D}_{A B}\right)+\left(E_{A}^{\mathrm{KS}}\left(\mathbb{D}_{A}\right)-E_{A}^{\mathrm{HF}}\left(\mathbb{D}_{A}\right)\right)+\left(E_{B}^{\mathrm{KS}}\left(\mathbb{D}_{B}\right)-E_{B}^{\mathrm{HF}}\left(\mathbb{D}_{B}\right)\right),
$$

where monomers' density matrices, $\mathbb{D}_{A}$ and $\mathbb{D}_{B}$, are obtained by converging Eq. (2). $E^{\mathrm{HF}}$ and $E^{\mathrm{KS}}$ are Hartree-Fock and Kohn-Sham energies. The KS energy is evaluated as

$$
E_{A}^{\mathrm{KS}}\left(\mathbb{D}_{A}\right)=\operatorname{Tr}\left(\mathbb{D}_{A}\left(2\left(\mathbb{T}+\mathbb{U}_{A}^{\mathrm{ne}}\right)+\mathbb{J}_{A}\right)\right)+\int_{\mathbb{R}^{3}} \epsilon_{\mathrm{xc}}\left(\rho_{A}(\mathbf{r}) ;\left\{\nabla \rho_{A}(\mathbf{r}) ; \ldots\right\}\right) \rho_{A}(\mathbf{r}) d^{3} \mathbf{r}+U_{A}^{\mathrm{nn}},
$$

where $U_{A}^{\mathrm{nn}}$ denotes the nuclear repulsion energy and $\epsilon_{\mathrm{xc}}$ is the exchange-correlation energy density. Substituting Hartree-Fock exchange for the KS exchange-correlation term in the above expression yields $E_{A}^{\mathrm{HF}}$.

The solution of Eq. (2) is performed in a freeze-and-thaw manner, ${ }^{33}$ that is, at a given step a density of one monomer is optimized with the density of the other monomer frozen. From the numerical point of view, the difference between our approach and the approach of Wesołowski and Weber ${ }^{33}$ is that we enforce orthogonality of the monomers' occupied orbitals, and the potential of non-additive kinetic energy does not appear in our model. In 
order to efficiently converge Eq. (2) in a self-consistent field (SCF) manner, and to minimize the number of intermediate matrices, the iterative solution of Eq. (2) is carried out with full non-additivity of the xc potential, i.e. without the approximation of Eq. (8). Thus, in Eq. (2) the KS matrix of the whole $A B$ system is present in each of two monomers' equations and Eq. (2) becomes a mere rearrangement of the KS equation for the dimer. The rationale behind such a procedure is as follows. If we start the freeze-and-thaw iterations from isolated monomers' densities slightly changed due to initial symmetric orthogonalization of the occupied orbitals, and then continuously deform each monomer's density in the field of its partner (keeping the orthogonality constraint), we converge to the orbitals which are well localized on the respective monomer. They can be interpreted as orbitals distorted by the intermolecular interaction. The above scheme is essentially identical to the Hartree-Fock Pauli blockade approach of Gutowski and Piela, ${ }^{32}$ who used equation analogous to Eq. (2) (with the dimer Fock matrix present in each of the monomer equations) to meaningfully decompose the interaction energy. In our scheme, upon convergence, the localized orbitals are utilized in Eq. (10) to yield dispersion-free energy of the dimer. As the dispersion-free energy of Eq. (10) is not invariant with respect to the unitary transformation mixing $A$ and $B$ orbitals, the localization of orbitals on monomers is indispensable in our approach. In principle, post-SCF localization methods ${ }^{34,35}$ could be used, however, the conceptual simplicity and computational scaling favors our approach of converging orbitals while keeping them localized on the monomers. Finally, the total interaction energy is obtained by combining $\mathcal{E}_{A B}^{\mathrm{PB}}$ with the dispersion contribution:

$$
\begin{aligned}
E_{\mathrm{int}}^{\mathrm{PB}+\mathrm{d}} & =\mathcal{E}_{A B}^{\mathrm{PB}}-E_{A}^{\mathrm{KS}}-E_{B}^{\mathrm{KS}}+E_{\mathrm{disp}}= \\
& =\mathcal{E}_{\mathrm{int}}^{\mathrm{PB}}+E_{\mathrm{disp}}
\end{aligned}
$$

where $E_{A}^{\mathrm{KS}}$ and $E_{B}^{\mathrm{KS}}$ are KS energies of isolated monomers.

\section{ALGORITHMIC DETAILS}

In this Section we derive and discuss an algorithm that solves Eq. (2). The new scheme alleviates the convergence problems of previously reported penalty function approach. ${ }^{22,23,32}$ In our experience the original penalty function approach to PB method suffers from a divergent and oscillatory behavior of the SCF iterations in many cases, e.g., for most of the 
dipole-interaction and charge transfer complexes reported in this work. The new algorithm presented below has convergence properties similar to those of standard single point KohnSham calculations for the $A B$ dimer.

In order to optimize occupied orbitals of one monomer in the presence of its counterpart, we adopt an exponential ansatz of orbital rotation,

$$
\mathbb{C}_{\rho, \text { new }}=\exp \left(-\mathbb{X}_{A}\right) \mathbb{C}_{\rho} .
$$

Anti-symmetric matrix $\mathbb{X}_{A}$ is defined as

$$
\mathbb{X}_{A}=\sum_{\substack{i \in A \\ a}} X_{i a}^{A}\left(\mathbb{C}_{i} \mathbb{C}_{a}^{\dagger}-\mathbb{C}_{a} \mathbb{C}_{i}^{\dagger}\right),
$$

where $i$ and $a$ denote occupied and virtual orbitals, respectively. It follows from Eq. (14) that $X_{i a}^{A}$ values determine the change of the occupied orbitals of monomer $A$ due to interaction with monomer $B$. In the following, we will focus on how to obtain $X_{i a}^{\zeta}$ parameters variationally. As occupied orbitals localized at monomer $B$ are excluded from summation in Eq. (15), the orthogonality between the occupied orbitals of $A$ and $B$ is maintained through the orbital rotation after it has been established in the zeroth iteration of Eq. (2). An iterative solution of Eq. (2) may proceed as follows:

1. Generate a set of orthogonal occupied orbitals by symmetric orthogonalization of converged occupied MO vectors of isolated monomers. Compute virtual orbitals as a complement to the occupied space.

2. Perform several microiterations to optimize occupied orbitals of monomer A interacting with monomer B. Rotate virtual orbitals of the whole system along with the occupied orbitals:

$$
\begin{aligned}
\mathbb{C}_{\text {occ }, A, \text { new }} & =\exp \left(-\mathbb{X}_{A}\right) \mathbb{C}_{\text {occ }, A}, \\
\mathbb{C}_{\text {virt,new }}^{\prime} & =\exp \left(-\mathbb{X}_{A}\right) \mathbb{C}_{\text {virt }} .
\end{aligned}
$$

3. Analogously to the above, optimize occupied orbitals of monomer B interacting with monomer A:

$$
\begin{aligned}
\mathbb{C}_{\text {occ }, B, \text { new }} & =\exp \left(-\mathbb{X}_{B}\right) \mathbb{C}_{\text {occ }, B}, \\
\mathbb{C}_{\text {virt,new }} & =\exp \left(-\mathbb{X}_{B}\right) \mathbb{C}_{\text {virt,new }}^{\prime}
\end{aligned}
$$


4. Go to step 2 if convergence is not achieved. Exit the loop if the density matrix error is acceptable.

Variational parameters contained in the $\mathbb{X}_{\zeta}$ matrix are determined iteratively through the Kohn-Sham total energy $\left(E^{\mathrm{KS}}\right)$ minimization. We cast the augmented Roothaan-Hall master equation $^{36,37}$ for the monomer $\mathrm{A}$,

$$
\left(\tilde{\mathbb{F}}_{n}^{v v}-\tilde{\mathbb{F}}_{n}^{o o}\right) \mathbb{X}+\mathbb{X}\left(\tilde{\mathbb{F}}_{n}^{v v}-\tilde{\mathbb{F}}_{n}^{o o}\right)+\sum_{i j}^{n-1}\left(\tilde{\mathbb{F}}_{i n}^{o v}-\tilde{\mathbb{F}}_{i n}^{v o}\right) T_{i j}^{-1} \operatorname{Tr}\left(\mathbb{D}_{j n}\left[\mathbb{D}_{n}, \mathbb{X}\right]\right)=\tilde{\mathbb{F}}_{n}^{v o}-\tilde{\mathbb{F}}_{n}^{o v}
$$

in a form that is suitable for restricting the variational space:

$$
\begin{aligned}
& \frac{\partial E^{\mathrm{KS}}(\mathbb{X})}{\partial X_{i a}}=0=4\left(\tilde{\mathbb{F}}_{n}\right)_{i a}+4 X_{i a}\left(e_{a}-e_{i}\right)+8 \sum_{k l}^{n-1}\left(\tilde{\mathbb{F}}_{k n}\right)_{i a} T_{k l}^{-1} \sum_{i^{\prime} a^{\prime}} X_{i^{\prime} a^{\prime}}\left(\mathbb{D}_{l}\right)_{i^{\prime} a^{\prime}} \\
& \text { where } \\
& \tilde{\mathbb{F}}=\mathbb{F}_{A}+\Delta \mathbb{V}_{A}^{\mathrm{xc}}+\mathbb{J}_{B} \\
& \tilde{\mathbb{F}}_{k l}=\tilde{\mathbb{F}}_{k}-\tilde{\mathbb{F}}_{l} \\
& T_{k l}=\operatorname{Tr}\left(\mathbb{D}_{k n}^{\dagger} \mathbb{D}_{l n}\right)
\end{aligned}
$$

For clarity, we drop the $A$ index at $\tilde{\mathbb{F}}, \mathbb{D}$, and $\mathbb{X}$. The notation introduced in Ref. 37 is used in Eq. (20). Here, $\tilde{\mathbb{F}}_{k}$ and $\mathbb{D}_{k}$ are Kohn-Sham and the density matrices, respectively, from the $k$-th iteration. Eq. (21) is written in the basis in which occupied-occupied and virtualvirtual blocks of the $\tilde{\mathbb{F}}$ matrix are diagonal. Such vectors can be computed by solving a pair of eigenvalue problems for occupied-occupied and virtual-virtual blocks of the KS matrix,

$$
\begin{aligned}
\left(\mathbb{C}_{\text {occ }, n}^{\dagger} \tilde{\mathbb{F}}_{n} \mathbb{C}_{\text {occ }, n}\right) \tilde{\mathbb{C}}_{i} & =e_{i} \tilde{\mathbb{C}}_{i} \quad(i \in A), \\
\left(\mathbb{C}_{\text {virt }, n}^{\dagger} \tilde{\mathbb{F}}_{n} \mathbb{C}_{\text {virt }, n}\right) \tilde{\mathbb{C}}_{a} & =e_{a} \tilde{\mathbb{C}}_{a}
\end{aligned}
$$

and transforming eigenvectors to the OAO basis:

$$
\begin{aligned}
\mathbb{C}_{i} & =\mathbb{C}_{\text {occ }, n} \tilde{\mathbb{C}}_{i} \\
\mathbb{C}_{a} & =\mathbb{C}_{\text {virt }, n} \tilde{\mathbb{C}}_{a}
\end{aligned}
$$

During the microiterations optimizing the A monomer orbitals we solve Eq. (21) with the condition $X_{i a}=0$ for $i \in B$, that is, we substitute the matrix of Eq. (15). This is necessary to prevent the orbitals of monomer $A$ from mixing with the occupied orbitals of monomer $B$. 
To facilitate solving Eq. (21), we define the intermediates:

$$
\begin{aligned}
X_{i a}^{(0)} & =-\frac{\left(\tilde{\mathbb{F}}_{n}\right)_{i a}}{e_{a}-e_{i}} \\
\sigma_{l}(\mathbb{X}) & =-2 \sum_{i a} X_{i a}\left(\mathbb{D}_{l}\right)_{i a} .
\end{aligned}
$$

With the new definitions, Eq. (21) can be reformulated as

$$
X_{i a}=X_{i a}^{(0)}+\frac{1}{e_{a}-e_{i}}\left(\sum_{k l}\left(\tilde{\mathbb{F}}_{k n}\right)_{i a} T_{k l}^{-1} \sigma_{l}(\mathbb{X})\right) .
$$

We can determine $\sigma_{l}(\mathbb{X})$ on the RHS of Eq. (32) if both sides of Eq. (32) are multiplied by $-2\left(\mathbb{D}_{m}\right)_{i a}$ and summed over $i, a$ :

$$
\begin{aligned}
\sigma_{m}(\mathbb{X}) & =\sigma_{m}\left(\mathbb{X}^{(0)}\right)+\sum_{k l} \tau_{k m} T_{k l}^{-1} \sigma_{l}(\mathbb{X}) \\
\tau_{k m} & =-\sum_{i a} \frac{2}{e_{a}-e_{i}}\left(\tilde{\mathbb{F}}_{k n}\right)_{i a}\left(\mathbb{D}_{m}\right)_{i a}
\end{aligned}
$$

Eq. (33) can be rearranged into the form of a $(n-1) \times(n-1)$ linear equation:

$$
\begin{aligned}
\sum_{l} \Omega_{m l} \sigma_{l}(\mathbb{X}) & =\sigma_{m}\left(\mathbb{X}^{(0)}\right) \quad(m=1, \ldots, n-1), \\
\Omega_{m l} & =\delta_{m l}-\sum_{k} \tau_{k m} T_{k l}^{-1} .
\end{aligned}
$$

In our implementation the maximum value of $n$ equals 8. Matrix exponential in Eq. (14) can be conveniently computed by squaring and scaling method:

$$
\begin{aligned}
\exp (\mathbb{A}) & \approx\left[T_{k}\left(\mathbb{A} / 2^{j}\right)\right]^{2^{j}}, \\
T_{k}(\mathbb{A}) & =\sum_{i=0}^{k} \mathbb{A}^{i} / i !
\end{aligned}
$$

Optimal $(j, k)$ pairs for a given norm of matrix $A$ were tabulated by Moler and Van Loan. ${ }^{38}$

\section{RESULTS AND DISCUSSION}

In order to assess the applicability of the dispersion-free approximation within the PB framework and to compare its results with the currently available benchmarks, we employed the database of noncovalent dimers of Zhao and Truhlar ${ }^{30,31}$. Their database gathers inter-

acting molecules in subsets according to the dominant character of the interaction. For the 
cases in which the relative discrepancy between the $\mathrm{PB}+$ dispersion and $\mathrm{CCSD}(\mathrm{T})$ results is noticeable, we additionally performed the symmetry-adapted perturbation theory (SAPT) calculations. All SAPT calculations reported in this work are accurate through the second order in the intermolecular interaction operator [see Eq. (39)]. They were performed with the Molpro package. ${ }^{39}$ Subroutines to carry out the PB calculations can be obtained directly from the authors.

\section{A. SAPT Overview}

The SAPT method allows one to represent the interaction energy as the sum of physically meaningful terms of the intermolecular perturbation theory: ${ }^{40}$

$$
E_{\text {int }}^{\mathrm{SAPT}}=E_{\text {elst }}^{(1)}+E_{\text {exch }}^{(1)}+E_{\text {ind }}^{(2)}+E_{\text {exch-ind }}^{(2)}+E_{\text {disp }}^{(2)}+E_{\text {exch-disp }}^{(2)} .
$$

The first-order terms on the RHS of Eq. (39) are the electrostatic and exchange energies, and the second-order contributions include induction, exchange-induction, dispersion, and exchange-dispersion energies, respectively. The method basically comes in two variants.

In the Hartree-Fock SAPT [SAPT $(\mathrm{HF})]$ the monomers are described by uncorrelated HF orbitals, i.e., at the zeroth-order with respect to the intramonomer correlation operator. This is denoted customarily by introducing the second superscript at the contributions to interaction energy, e.g. $E_{\text {disp }}^{(20)}$.

In the DFT-based SAPT [SAPT(DFT)], ${ }^{17,41,42}$ the monomers are described by the KS orbitals which are presumed to include the intramonomer correlation. The second-order terms are obtained from the coupled KS (CKS) theory. The SAPT(DFT) interaction energy of Eq. (39) is often augmented by the residual HF term defined as

$$
\delta_{\mathrm{HF}}=E_{\mathrm{int}}^{\mathrm{HF}}-\left(E_{\mathrm{elst}}^{(10)}+E_{\mathrm{exch}}^{(10)}+E_{\mathrm{ind}}^{(20)}+E_{\text {exch-ind }}^{(20)}\right)
$$

with $E_{\text {int }}^{\mathrm{HF}}$ being the supermolecular HF interaction energy. The purpose of the $\delta_{\mathrm{HF}}$ term is to account for higher-order induction effects and other residual terms necessary to obtain a convergent result. Thus, the full SAPT(DFT) interaction energy is

$$
E_{\text {int }}^{\mathrm{SAPT}(\mathrm{DFT}) \delta}=E_{\text {int }}^{\mathrm{SAPT}(\mathrm{DFT})}+\delta_{\mathrm{HF}}
$$




\section{B. Computational Details}

Both $\mathrm{PB}$ and the cited reference calculations were performed with with counterpoise correction. The interaction energies do not include contributions from deformation of monomers. Reference $\operatorname{CCSD}(\mathrm{T})$ interaction energies and SAPT(DFT) dispersion contributions,

$$
E_{\text {disp }}^{\mathrm{SAPT}(\mathrm{DFT})}=E_{\text {disp }}^{(2)}+E_{\text {exch-disp }}^{(2)}
$$

are taken from Ref. 21. They were computed using the aug-cc-pVTZ basis set with midbond functions. The dispersion energy in Eq. (13) is either taken from SAPT(DFT):

$$
E_{\text {int }}^{\mathrm{PB}+\mathrm{d}}=\mathcal{E}_{\text {int }}^{\mathrm{PB}}+E_{\text {disp }}^{\mathrm{SAPT}(\mathrm{DFT})}
$$

or from the numerical fit to the dispersion and exchange-dispersion terms of Ref. 43,

$$
E_{\text {int }}^{\mathrm{PB}+\mathrm{df}}=\mathcal{E}_{\text {int }}^{\mathrm{PB}}+E_{\text {disp }}^{\mathrm{fit}}
$$

$E_{\text {int }}^{\mathrm{KS}}$ stands for the supermolecular KS interaction energy.

The SAPT values in Table VI are computed in aug-cc-pVTZ basis set with the asymptoticallycorrected PBE0 functional. $\mathrm{SAPT}(\mathrm{DFT}) \delta$ interaction energies include $\delta_{\mathrm{HF}}$ which is also listed separately.

\section{Noncovalent interactions database}

\section{1. $\mathrm{HB} 6 / 04$}

The results for six complexes form the HB6/04 database are presented in Table I. In the assessment of the dispersion-free approach $\left(\mathcal{E}_{\text {int }}^{\mathrm{PB}}\right)$ in combination with two formulations of dispersion energy (see Sec. IV B) is compared with the supermolecular interaction energies $E_{\text {int }}^{\mathrm{KS}}$ and $E_{\text {int }}^{\mathrm{CCSD}(\mathrm{T})}$. The $\mathcal{E}_{\text {int }}^{\mathrm{PB}}$ values show practically no dependence on the basis set, aug-cc-pVDZ vs. aug-cc-pVTZ and only a weak dependence on the type of the hybrid functional, PBE0 vs. B3LYP. The difference between $E_{\text {int }}^{\mathrm{KS}}$ and $\mathcal{E}_{\text {int }}^{\mathrm{PB}}$ arises from the different xc potential acting between the interacting monomers: the same as the intramonomer potential in the former and the exact exchange in the latter. The differences between the two quantities are considerable and roughly similar (but not equal) to the dispersion energy. For the 
first four complexes $\mathrm{PB}+\mathrm{d}$ or $\mathrm{PB}+\mathrm{df}$ are in excellent agreement with the $\mathrm{CCSD}(\mathrm{T})$ benchmark. For the more strongly-bound complexes with double hydrogen bonds as $(\mathrm{HCOOH})_{2}$ the agreement deteriorates to the $20 \%$ overbinding.

\section{2. $C T 7 / 04$}

The group of charge-transfer complexes consists of donor-acceptor pairs ordered according to increasing strength of interaction. The dispersion-free energies, $\mathcal{E}_{\text {int }}^{\mathrm{PB}}$, are always above the total reference interaction energies. However, after the addition of dispersion contribution, severe overbinding is present except for the weak complexes involving $\mathrm{F}_{2}$.

For the complexes of $\mathrm{ClF}$ (particularly $\mathrm{NH}_{3} \cdots \mathrm{ClF}$ ) the discrepancy between $\mathrm{PB}+\mathrm{d}$ $(\mathrm{PB}+\mathrm{df})$ and $\mathrm{CCSD}(\mathrm{T})$ reaches $55 \%(\mathrm{~B} 3 \mathrm{LYP})$ to over $70 \%(\mathrm{PBE} 0)$. The question is whether the reason for this behavior is the failure of the PB method for strongly overlapping monomers, or the problem with xc potentials which fail to properly match the donor-acceptor properties of subsystems. This isse is discussed in more detail below in Sec. IV C 7 .

\section{3. $\mathrm{DI} 6 / 04$}

The group of molecular complexes in this database is primarily bound by the dispersion energy as $\mathcal{E}_{\text {int }}^{\mathrm{PB}}$ represents only a fraction of the total interaction energy. The $\mathrm{PB}+\mathrm{d}(\mathrm{df})$ treatment faithfully represents the bonding trends of this group as compared to $\operatorname{CCSD}(\mathrm{T})$ with a slight overbinding of about $10-15 \%$ (with one outlier, $\mathrm{CH}_{3} \mathrm{SH} \cdots \mathrm{HCl}$, with $25 \%$ overbinding).

\section{WIY $/ 05$}

In this group of van der Waals complexes $\mathcal{E}_{\text {int }}^{\mathrm{PB}}$ predicts all interactions to be repulsive in both functionals. This is correct behavior expected from a dispersion-free approximation. In combination with the dispersion energy, the $\mathrm{PB}+\mathrm{d}(\mathrm{df})$ approach yields the interaction energies in excellent agreement with $\operatorname{CCSD}(\mathrm{T})$. It should be pointed out that the supermolecular $E_{\text {int }}^{\mathrm{KS}}$ results obtained in PBE0 and B3LYP show trends that are opposite to one another.

This is in striking contrast to $\mathcal{E}_{\text {int }}^{\mathrm{PB}}$ which remains virtually independent of the functional. 


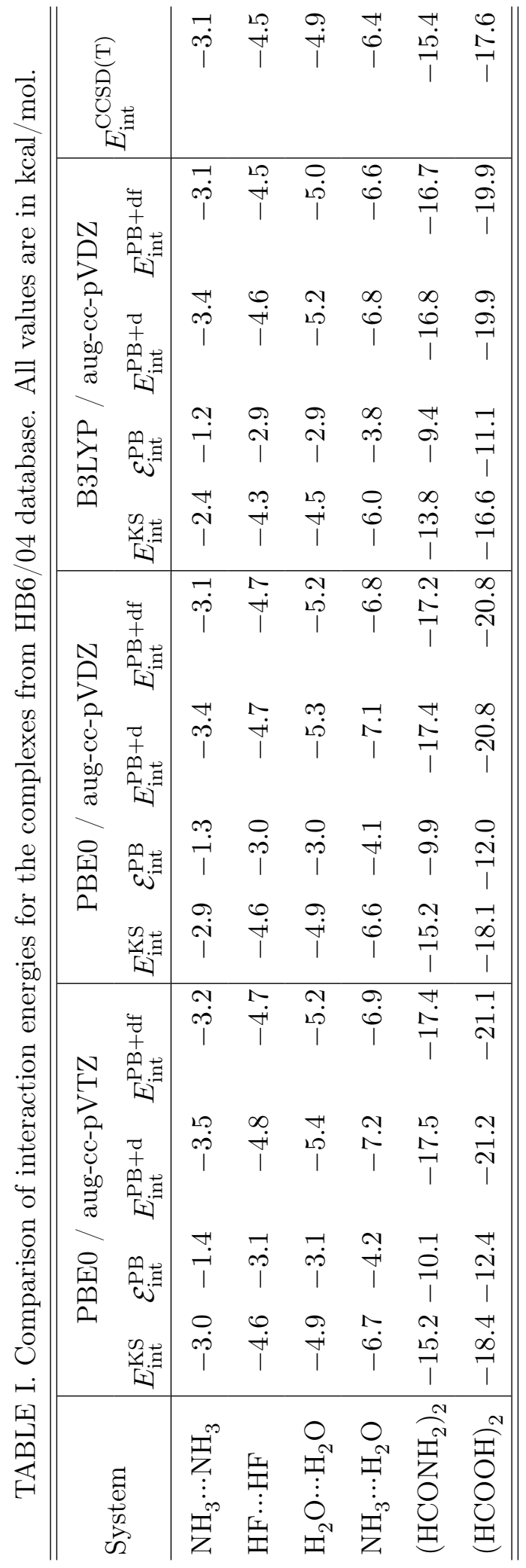




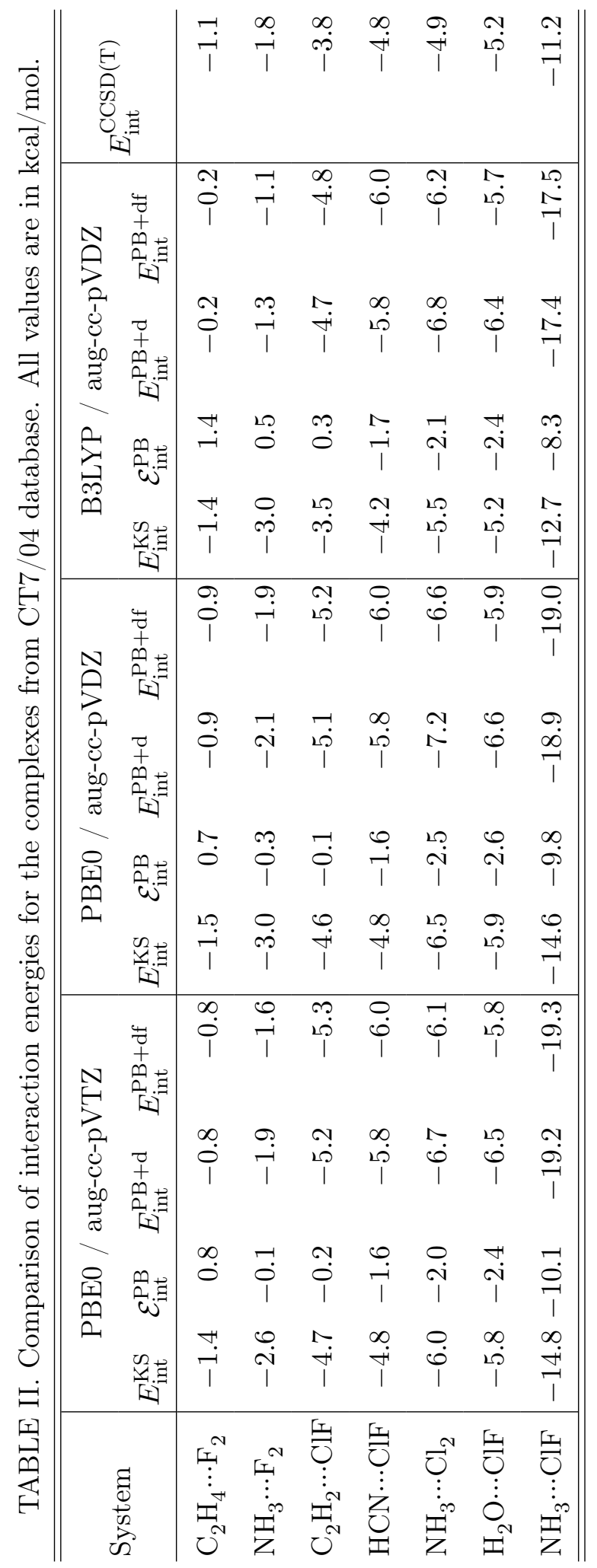




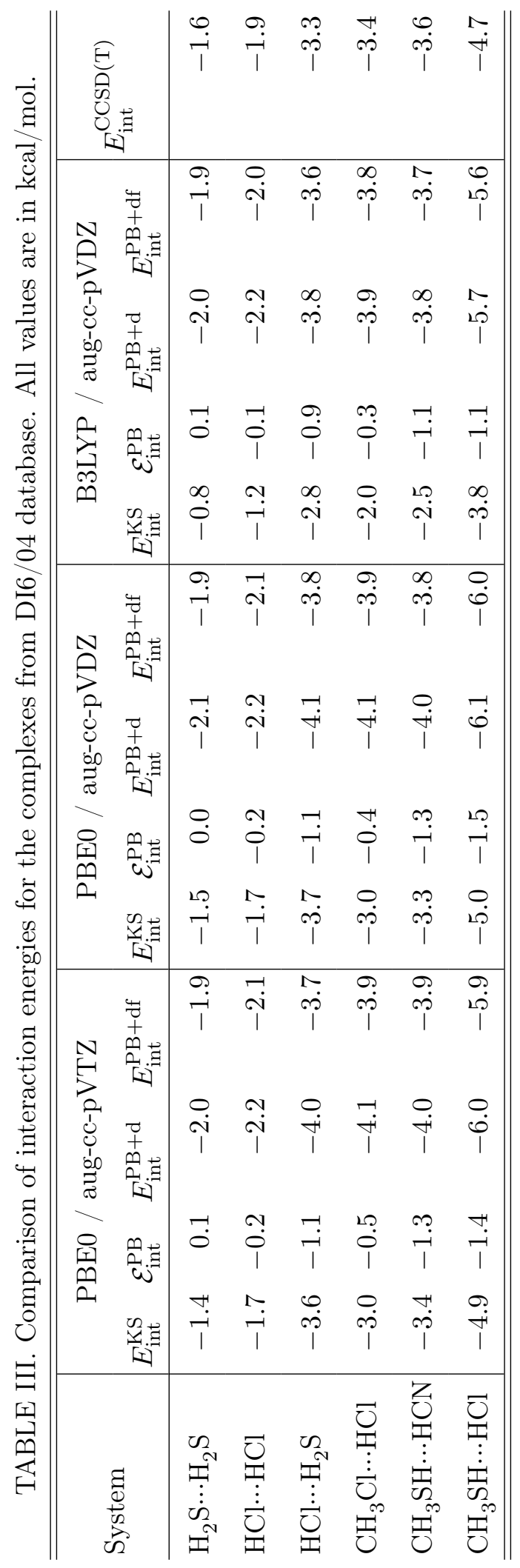


We note that similar behavior of the dispersion-free energy with respect to the functional was observed by Kamiya, Tsuneda, and Hirao ${ }^{20}$.

\section{5. $P P S 5 / 05$}

This database contains representative $\pi$-stacking complexes. The dispersion free approximation yields strongly repulsive interaction energies for all but one of the complexes. The repulsive interactions for the three conformers of benzene dimer show consistency between two functionals PBE0 and B3LYP and two different basis sets: two stacking configurations, sandwich and displaced have equal interactions and the T-shaped one is less repulsive. When combined with the dispersion energy, $\mathrm{PB}+\mathrm{d}(\mathrm{df})$ follows closely the $\mathrm{CCSD}(\mathrm{T})$ benchmarks. The slight overbinding of about $10 \%$ still persists. Unlike for the HB6/04 complexes the $E_{\text {int }}^{\mathrm{KS}}$ interaction energies are in poor agreement with both $\mathrm{PB}+\mathrm{d}(\mathrm{df})$ and the benchmarks. The ordering of the three conformers of the benzene dimer is wrong in both functionals.

\section{Comparison with SAPT and DFT-based approaches}

In Table VI the results of the $\mathrm{PB}+\mathrm{d}$ approach are compared with two versions of the second-order SAPT: one based on the HF description of monomers [SAPT(HF)] and one based on the DFT monomer description $[\mathrm{SAPT}(\mathrm{DFT}) \delta]$. As described in Sec. IV A, $\mathrm{SAPT}(\mathrm{DFT}) \delta$ incorporates the HF-derived residual term, $\delta_{\mathrm{HF}}$, into the interaction energy. For the comparison we chose three problematic cases discussed above $(\mathrm{HCOOH})_{2}, \mathrm{NH}_{3} \ldots \mathrm{ClF}$, $\mathrm{CH}_{3} \mathrm{SH} \cdots \mathrm{HCl}$, as well as $\mathrm{Ne}_{2}$ and $\mathrm{T}$-shaped $\left(\mathrm{C}_{6} \mathrm{H}_{6}\right)_{2}$ which are notoriously difficult for theory. At the first glance SAPT(DFT) $\delta$ appears to provide much better agreement with the $\mathrm{CCSD}(\mathrm{T})$ benchmarks than $\mathrm{PB}+\mathrm{d}$ for $(\mathrm{HCOOH})_{2}, \mathrm{NH}_{3} \cdots \mathrm{ClF}, \mathrm{CH}_{3} \mathrm{SH} \cdots \mathrm{HCl}$. However, this agreement is contingent on including the $\delta_{\mathrm{HF}}$ term in the interaction energy. In these three complexes the $\delta_{\mathrm{HF}}$ is very large in magnitude and in $\mathrm{NH}_{3} \cdots \mathrm{ClF}$ it is responsible for the entire binding effect in $\mathrm{SAPT}(\mathrm{DFT}) \delta$. The large $\delta_{\mathrm{HF}}$ is in itself and indicator that the convergence of SAPT is problematic. ${ }^{44,45} \mathrm{In} \mathrm{Ne}_{2}$ and T-shaped benzene dimer the $\mathrm{PB}+\mathrm{d}$ approach is closer to $\operatorname{CCSD}(\mathrm{T})$. The performance of $\operatorname{SAPT}(\mathrm{HF})$ appears to be erratic. On the one hand, in $(\mathrm{HCOOH})_{2}$ it agrees reasonably well with $\operatorname{CCSD}(\mathrm{T})$; on the other hand, in both $\mathrm{NH}_{3} \cdots \mathrm{ClF}$ and in $\mathrm{CH}_{3} \mathrm{SH} \cdots \mathrm{HCl}$ it severely underbinds. 


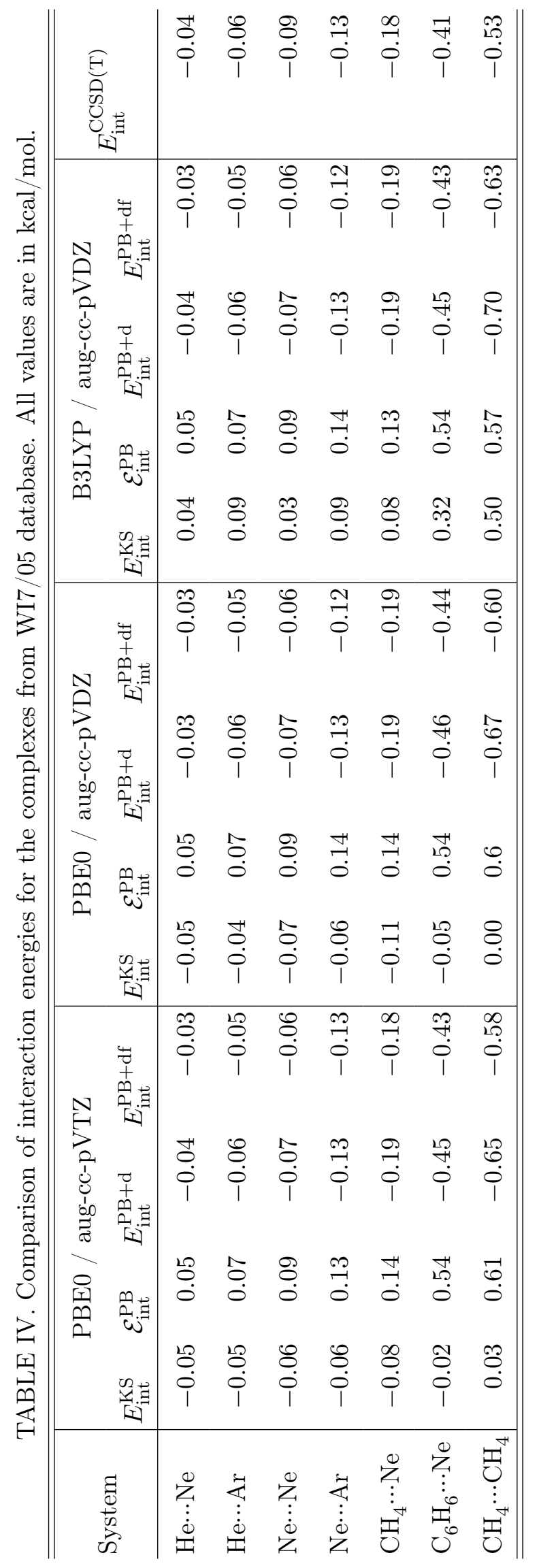




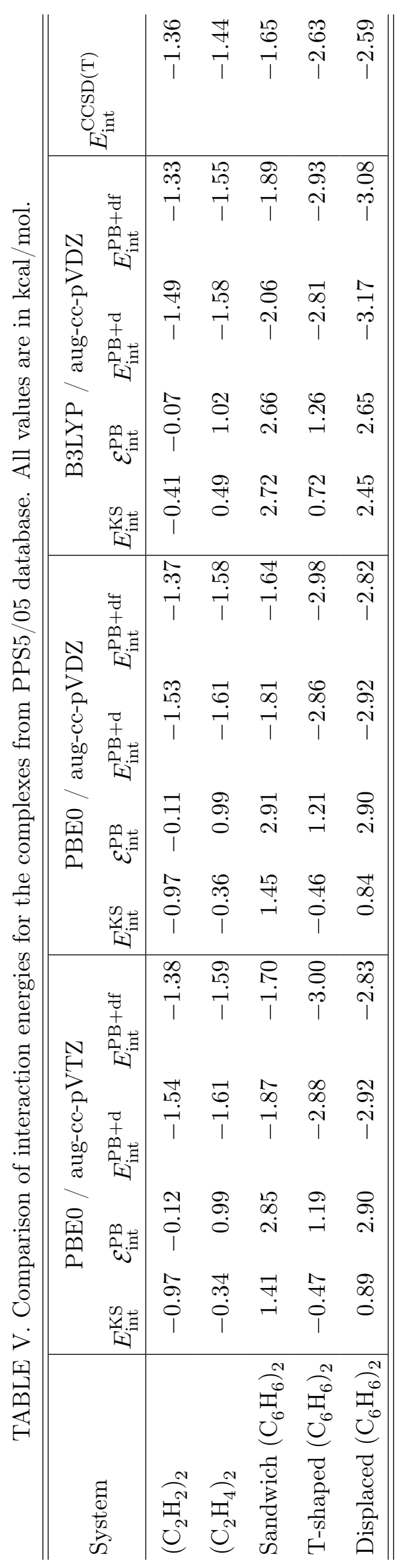


TABLE VI. Comparison of the $\mathrm{PB}+\mathrm{d}$ approach with $\mathrm{SAPT}(\mathrm{HF})$ and $\mathrm{SAPT}(\mathrm{DFT})$. The $\mathrm{PB}+\mathrm{d}$ values are obtained with intramonomer functional PBE0 while SAPT(DFT) terms are obtained with asymptotically corrected variant of $\mathrm{PBE} 0, \mathrm{PBE} 0 \mathrm{AC} . \delta_{\mathrm{HF}}$ denotes the residual $\mathrm{HF}$ terms (see the text). All values are in $\mathrm{kcal} / \mathrm{mol}$.

\begin{tabular}{lrrrrr}
\hline \hline Molecule & $E_{\text {int }}^{\mathrm{SAPT}(\mathrm{HF})}$ & $E_{\text {int }}^{\mathrm{SAPT}(\mathrm{DFT}) \delta}$ & $\delta_{\mathrm{HF}}$ & $E_{\text {int }}^{\mathrm{PB}+\mathrm{d}}$ & $E_{\text {int }}^{\mathrm{CCSD}(\mathrm{T})}$ \\
\hline$(\mathrm{HCOOH})_{2}$ & -16.35 & -16.72 & -6.47 & -21.17 & -17.60 \\
$\mathrm{NH}_{3} \cdots \mathrm{ClF}$ & -3.55 & -9.93 & -9.81 & -19.2 & -11.17 \\
$\mathrm{Ne}_{2}$ & -0.05 & -0.07 & -0.002 & -0.07 & -0.09 \\
$\mathrm{CH}_{3} \mathrm{SH} \cdots \mathrm{HCl}$ & -2.82 & -5.21 & -2.48 & -6.04 & -4.74 \\
T-shaped $\left(\mathrm{C}_{6} \mathrm{H}_{6}\right)_{2}$ & -2.46 & -2.38 & -0.31 & -2.88 & -2.63 \\
\hline \hline
\end{tabular}


In Tables VII the $\mathrm{PB}+\mathrm{d} / \mathrm{PBE} 0$ method is compared against other DFT approaches specifically tailored for intermolecular interactions. Clearly, $\mathrm{PB}+\mathrm{d}$ approach achieves high accuracy in WI7/05 and PPS5/05 sets when compared to other approximate methods, notably without additional parametrization. Its performance with respect to dispersion-dominated complexes is comparable to that of $\mathrm{dlDF}+\mathrm{D}$ and B97-D methods, which probably represent the best of what the contemporary well-parametrized functionals can achieve for these interactions. However, inspecting first three columns of data in Tables VII and VIII reveals that the $\mathrm{PB}+\mathrm{d}$ approximation worsens the results of the underlying functional (PBE0) and performs poorly compared to other approaches for the hydrogen-bonded and dipole-bound complexes. For the charge-transfer group all the approaches, except for dlDF $+\mathrm{D}$, appear to have problems.

\section{Charge-transfer interactions}

Let us examine more closely the case of charge-transfer interactions. Fig. 1 shows the dependence of $E_{\mathrm{int}}^{\mathrm{PB}}$ and $E_{\mathrm{int}}^{\mathrm{PB}+\mathrm{d}}$ in $\mathrm{NH}_{3} \cdots \mathrm{ClF}$ on the intersystem distance and the comparison with $\operatorname{CCSD}(\mathrm{T})$ as well as with the supermolecular $E_{\text {int }}^{\mathrm{KS}}$ term. The PB interaction energy curve has a well depth of about $10 \mathrm{kcal} / \mathrm{mol}$, which is ca. $5 \mathrm{kcal} / \mathrm{mol}$ less than the $E_{\text {int }}^{\mathrm{KS}}$ curve and about $4 \mathrm{kcal} / \mathrm{mol}$ more than the $E_{\mathrm{int}}^{\mathrm{HF}}$ curve. However, when combined with the dispersion energy from SAPT, $\mathrm{PB}+\mathrm{d}$ descends rapidly. Clearly, these two terms are incompatible. There are two reasons for this. First, because of a short intersystem distance, the PB approach already includes some dispersion effect (i.e. no longer serves as a dispersion-free approximation) and combining it with the SAPT-based dispersion leads to double counting. Second, $E_{\text {int }}^{\mathrm{KS}}$ PBE0 calculations (and to a lesser extent with B3LYP, see Table II) lead to a considerable overbinding, as evidenced by a $4 \mathrm{kcal} / \mathrm{mol}$ overestimation of the well depth of $E_{\text {int }}^{\mathrm{KS}}$ compared to $\operatorname{CCSD}(\mathrm{T})$ in Fig. 1.

We will demonstrate below, using PBE0 as an example, that this overbinding can be traced, at least partially, to the tendency of these functionals to make the donor molecule too good a donor and the acceptor molecule too good an acceptor of electrons. This problem has long been attributed ${ }^{51}$ to the mismatch between the HOMO energy of the donor and the LUMO energy of the acceptor leading to a too narrow fundamental gap in some hybrid functionals. Alternatively, this problem can be related to the delocatization error in the 


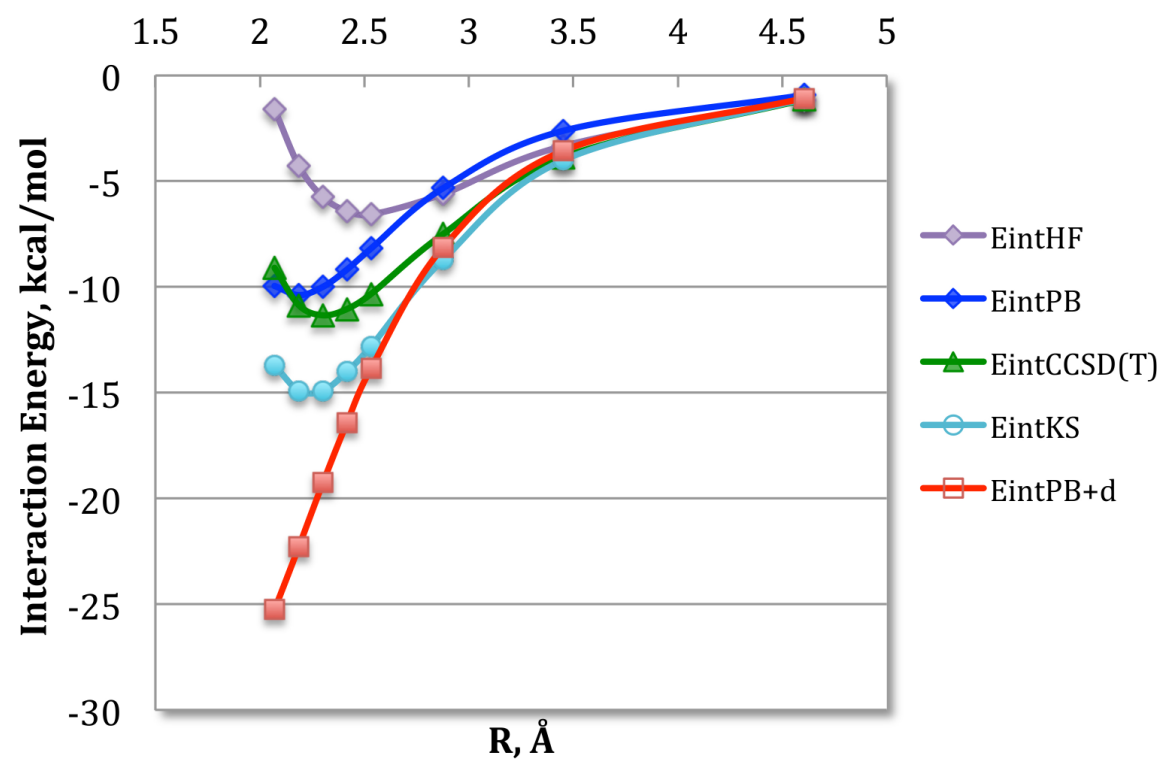

FIG. 1. The interaction energy curves for the $\mathrm{NH}_{3} \cdots \mathrm{ClF}$ complex. $R$ denotes the distance between $\mathrm{N}$ and $\mathrm{Cl}$. The $\mathrm{PB}$ and $\mathrm{KS}$ interaction energies are computed with the PBE0 functional and aug-cc-pVTZ basis set. The HF and CCSD(T) terms are computed with the aug-cc-pVQZ basis set.

approximate xc functionals. ${ }^{9}$ To detect the presence of the delocalization error we inspect the behavior of the total energy between the integer values of electron numbers. In the exact functional this dependence should be linear from the cation to the neutral and from the neutral to the anion, showing a discontinuity in the first derivative at the neutral. ${ }^{52}$ Departures from the linear behavior with respect to fractional electron numbers indicate the presence of the delocalization error. ${ }^{9,53,54}$ For the two constituent molecules $\mathrm{ClF}$ and $\mathrm{NH}_{3}$ we performed calculations of energy as a function of fractional electron numbers with the PBE0 functional. The calculations were performed with NWChem package. ${ }^{55}$ The longrange corrected PBE0 (LC-PBE0) is included for comparison. The results in Fig. 2 and 3 demonstrate that LC-PBE0 yields a linear relationship of the total energy with fractional electron numbers for both $\mathrm{ClF}$ and $\mathrm{NH}$, i.e., it behaves as the exact functional. For $\mathrm{NH}_{3}$ a positive slope of neutral-to-anion curve indicates that $\mathrm{NH}_{3}$ does not form a negative ion, unlike $\mathrm{ClF}$ which does. By contrast, PBE0 alone leads to convex dependence of energy with respect to fractional electron numbers for both molecules. This means that in the PBE0 functional ClF becomes more prone to accepting a fractional charge compared to the 
TABLE VII. Mean unsigned percentage error of DFT-based approaches. All results were obtained with aug-cc-pVTZ basis set. Results other than $\mathrm{PB}+\mathrm{d}$ or supermolecular PBE0 were taken from Ref. 43

\begin{tabular}{|c|c|c|c|c|c|}
\hline \multirow[b]{2}{*}{$\mathrm{PB}+\mathrm{d} / \mathrm{PBE} 0$} & \multicolumn{5}{|c|}{ HB6/04 CT7/04 DI6/04 WI7/05 PPS5/05 } \\
\hline & 12.5 & 31.4 & 20.4 & 9.4 & 12.2 \\
\hline $\mathrm{PBE} 0^{46}$ & 2.9 & 23.7 & 8.6 & 54.6 & 101.3 \\
\hline M06-2X ${ }^{47}$ & 4.3 & 12.5 & 5.3 & 90.4 & 22.9 \\
\hline $\mathrm{HFD}^{43,48,49}$ & 24.9 & 25.2 & 20.7 & 24.1 & 18.2 \\
\hline $\mathrm{dlDF}+\mathrm{D}^{21}$ & 4.5 & 1.7 & 5.5 & 12.9 & 11.4 \\
\hline $\mathrm{B} 97-\mathrm{D}^{50}$ & 7.2 & 59.7 & 6.1 & 36.0 & 3.1 \\
\hline
\end{tabular}

TABLE VIII. Mean unsigned error in kcal/mol. All results were obtained with aug-cc-pVTZ basis set. Results other than $\mathrm{PB}+\mathrm{d}$ or supermolecular PBE0 were taken from Ref. 43

\begin{tabular}{lccccc}
\hline & HB6/04 CT7/04 & DI6/04 & WI7/05 & PPS5/05 \\
\hline PB +d/PBE0 & 1.3 & 2.0 & 0.6 & 0.03 & 0.23 \\
PBE0 & 0.3 & 1.1 & 0.2 & 0.17 & 2.04 \\
M06-2X & 0.4 & 0.5 & 0.1 & 0.11 & 0.45 \\
HFD & 2.4 & 1.4 & 0.7 & 0.05 & 0.30 \\
dlDF+D & 0.2 & 0.1 & 0.1 & 0.02 & 0.21 \\
B97-D & 0.5 & 1.6 & 0.2 & 0.04 & 0.06 \\
\hline \hline
\end{tabular}




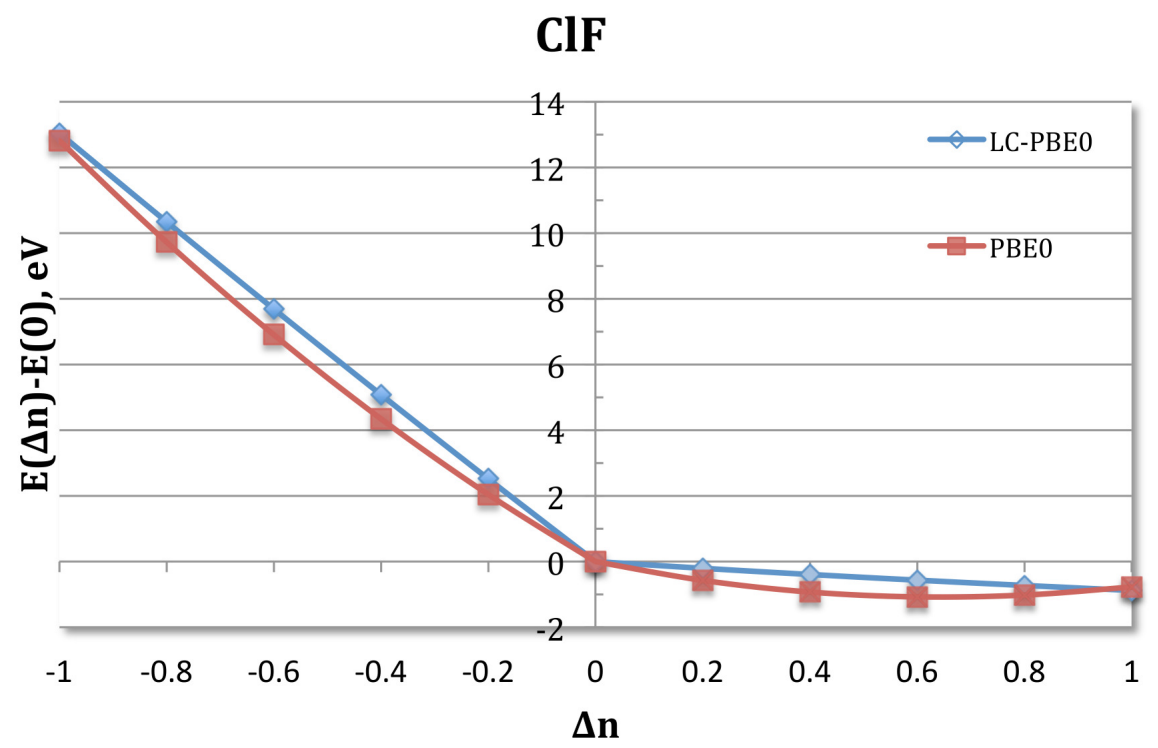

FIG. 2. Energy of $\mathrm{ClF}$ as a function of fractional electron number $\Delta n$ relative to the neutral system $(\Delta n=0)$. The LC-PBE0 functional employs the range separation parameter 0.3 , a portion of 0.25 $\mathrm{HF}$ exchange in the short range, and 1.0 HF exchange in the long range. All calculations are performed in aug-cc-pVDZ basis set.

exact functional. Similarly, $\mathrm{NH}_{3}$ is also more prone to loose a fractional charge compared to the exact functional. Since a CT complex $\mathrm{NH}_{3} \cdots \mathrm{ClF}$ involves partial donation of electron charge from $\mathrm{NH}_{3}$ to $\mathrm{ClF}$, the PBE0 functional's delocalization error artificially enhances both the ability of $\mathrm{NH}_{3}$ to donate and of $\mathrm{ClF}$ to accept the electron density. These enhanced donor-acceptor abilities are not correctable by the Pauli Blockade. They can be remedied by employing functionals which accurately describe fundamental gaps as well as show the correct linear dependence of total energy on fractional electron numbers.

\section{CONCLUSIONS}

In this work we presented comprehensive tests of recently-developed dispersion-free functional approach.

Except for dispersion-dominated complexes for which $\mathrm{PB}+\mathrm{d}$ yields excellent results, systematic overbinding has been observed. This error is most severe for strongly bound dimers with short intersystem distances where the distinction between the intrasystem and intersystem correlation effects becomes problematic. In these complexes combining the PB 


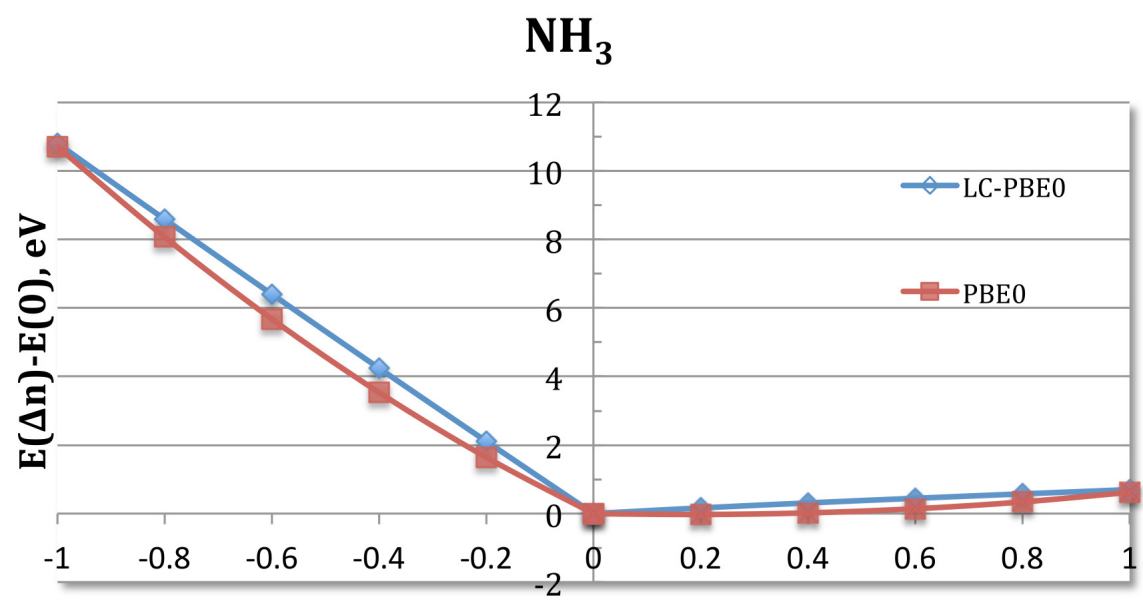

$\Delta \mathbf{n}$

FIG. 3. Energy of $\mathrm{NH}_{3}$ as a function of fractional electron number $\Delta n$ relative to the neutral system $(\Delta n=0)$. The LC-PBE0 functional employs the range separation parameter 0.3, a portion of $0.25 \mathrm{HF}$ exchange in the short range, and 1.0 $\mathrm{HF}$ exchange in the long range. All calculations are performed in aug-cc-pVDZ basis set.

interaction with the SAPT-based dispersion is bound to fail. In case of charge-transfer complexes, the systematic error of $\mathrm{PB}+\mathrm{d}$ methods adds to the same-sign error of the underlying functional. The analysis of the delocalization error of the PBE0 functional have indicated that the overbinding of charge-transfer complexes can be partially attributed to the erratic behavior of the approximate functional used in conjunction with $\mathrm{PB}+\mathrm{d}$ method.

The new iterative procedure for the solution of $\mathrm{PB}$ equations has been presented. It converges smoothly for cases where the originally proposed algorithm was not able to yield a converged solution. An exponential parametrization of monomer density matrices and their optimization with the algorithm of quadratic convergence allowed us to sidestep the poor convergence properties of penalty function of the original PB method.

\section{ACKNOWLEDGEMENTS}

This work was supported by the Polish Ministry of Science and Higher Education (Grant No. IP2010 034170) and by U.S. National Science Foundation (Grant No. 1152474). We also acknowledge computational resources of the Interdisciplinary Centre for Mathematical and Computational Modelling of the University of Warsaw. 


\section{REFERENCES}

${ }^{1}$ P. Hohenberg and W. Kohn, Phys. Rev. 155, B864 (1962).

${ }^{2}$ W. Kohn and L. J. Sham, Phys. Rev. 140, A1133 (1965).

${ }^{3}$ L. Goerigk and S. Grimme, Phys. Chem. Chem. Phys. 13, 6670 (2011).

${ }^{4}$ W. Koch and M. C. Holthausen, A Chemist's Guide to Density Functional Theory (WileyVCH Verlag GmbH, Weinheim, 2001).

${ }^{5}$ J. P. Perdew and S. Kurt, "A primer in density functional theory," (Springer Berlin / Heidelberg, 2003) Chap. Density Functionals for Non-relativistic Coulomb Systems in the New Century.

${ }^{6}$ S. Kristyán and P. Pulay, Chem. Phys. Lett. 229, 175 (1994).

${ }^{7}$ P. Hobza and J. Šponer, Chem. Rev. 99, 3247 (1999).

${ }^{8}$ M. Allen and D. Tozer, J. Chem. Phys. 117, 11113 (2002).

${ }^{9}$ A. J. Cohen, P. Mori-Sánchez, and W. Yang, Chem. Rev. 112, 289 (2012), http://pubs.acs.org/doi/pdf/10.1021/cr200107z.

${ }^{10}$ M. Dion, H. Rydberg, E. Schröder, D. C. Langreth, and B. I. Lundqvist, Phys. Rev. Lett. 92, 246401 (2004).

${ }^{11}$ T. Thonhauser, V. R. Cooper, S. Li, A. Puzder, P. Hyldgaard, and D. C. Langreth, Phys. Rev. B 76, 125112 (2007).

${ }^{12}$ O. Vydrov and T. Van Voorhis, Phys. Rev. Lett. 103, 063004 (2009).

${ }^{13}$ O. A. von Lilienfeld, I. Tavernelli, U. Rothlisberger, and D. Sebastiani, Phys. Rev. Lett. 93, 153004 (2004).

${ }^{14}$ X. Wu, M. C. Vargas, S. Nayak, V. Lotrich, and G. Scoles, J. Chem. Phys. 115, 8748 (2001).

${ }^{15}$ S. Grimme, J. Antony, S. Ehrlich, and H. Krieg, J. Chem. Phys. 132, 154104 (2010).

${ }^{16}$ A. D. Becke and E. R. Johnson, J. Chem. Phys. 122, 154104 (2005).

${ }^{17}$ A. J. Misquitta, B. Jeziorski, and K. Szalewicz, Phys. Rev. Lett. 91, 033201 (2003).

${ }^{18}$ A. Heßelmann and G. Jansen, Chem. Phys. Lett. 367, 778 (2003).

${ }^{19}$ L. A. Burns, Á. Vázquez-Mayagoitia, B. G. Sumpter, and C. D. Sherrill, J. Chem. Phys. 134, 084107 (2011).

${ }^{20}$ M. Kamiya, T. Tsuneda, and K. Hirao, J. Chem. Phys. 117, 6010 (2002). 
${ }^{21}$ K. Pernal, R. Podeszwa, K. Patkowski, and K. Szalewicz, Phys. Rev. Lett. 103, 263201 (2009).

${ }^{22}$ Ł. Rajchel, P. Żuchowski, M. Szczęśniak, and G. Chałasiński, Phys. Rev. Lett. 104, 163001 (2010).

${ }^{23}$ Ł. Rajchel, P. Żuchowski, M. Szczęśniak, and G. Chałasiński, Chem. Phys. Lett. 486, 160 (2010).

${ }^{24}$ H. Iikura, T. Tsuneda, T. Yanai, and K. Hirao, J. Chem. Phys. 115, 3540 (2001).

${ }^{25}$ P. Nozières and D. Pines, Phys. Rev. 111, 442 (1958).

${ }^{26}$ H. Stoll and A. Savin, in Density Functional Methods in Physics, edited by R. M. Dreizler and J. d. Providencia (Plenum, New York, 1985) p. 177.

${ }^{27}$ I. Panas, Chem. Phys. Lett. 245, 171 (1995).

${ }^{28}$ R. D. Adamson, J. P. Dombroski, and P. M. W. Gill, Chem. Phys. Lett. 254, 329 (1996).

${ }^{29}$ R.-F. Liu, C. A. Franzese, R. Malek, P. S. Zuchowski, J. G. Angyan, M. M. Szczesniak, and G. Chalasinski, Journal of Chemical Theory and Computation 7, 2399 (2011), http://pubs.acs.org/doi/pdf/10.1021/ct200243s.

${ }^{30}$ Y. Zhao and D. Truhlar, J. Phys. Chem. A 109, 5656 (2005).

${ }^{31}$ Y. Zhao and D. Truhlar, J. Chem. Theory Comput. 1, 415 (2005).

${ }^{32}$ M. Gutowski and L. Piela, Mol. Phys. 64, 337 (1988).

${ }^{33}$ T. Wesołowski and J. Weber, Chem. Phys. Lett. 248, 71 (1996).

${ }^{34}$ J. Pipek and P. Mezey, J. Chem. Phys. 90, 4916 (1989).

${ }^{35}$ D. Kleier, T. Halgren, J. Hall Jr, and W. Lipscomb, J. Chem. Phys. 61, 3905 (1974).

${ }^{36}$ S. Høst, J. Olsen, B. Jansík, L. Thøgersen, P. Jørgensen, and T. Helgaker, J. Chem. Phys. 129, 124106 (2008).

${ }^{37}$ S. Høst, B. Jansík, J. Olsen, P. Jørgensen, S. Reine, and T. Helgaker, Phys. Chem. Chem. Phys. 10, 5344 (2008).

${ }^{38}$ C. Moler and C. Van Loan, SIAM Review 45, 3 (2003).

${ }^{39}$ H.-J. Werner, P. J. Knowles, F. R. Manby, M. Schütz, et al., "Molpro, version 2010.1, a package of ab initio programs," (2010).

${ }^{40}$ B. Jeziorski, R. Moszyński, and K. Szalewicz, Chem. Rev. 94, 1887 (1994).

${ }^{41}$ A. J. Misquitta and K. Szalewicz, Chem. Phys. Lett. 357, 301 (2002).

${ }^{42}$ G. Jansen and A. Heßelmann, J. Phys. Chem. A 105, 11156 (2001). 
${ }^{43}$ R. Podeszwa, K. Pernal, K. Patkowski, and K. Szalewicz, J. Phys. Chem. Lett. 1, 550 (2010).

${ }^{44}$ D. N. Snyder, M. M. Szczęśniak, and G. Chałasiński, J. Chem. Phys. 130, 224704 (2009).

${ }^{45}$ R.-F. Liu, C. Franzese, R. Malek, P. S. Żuchowski, J. G. Ángyán, M. M. Szczęśniak, and G. Chałasiński, J. Chem. Theory Comput. 7, 2399 (2011).

${ }^{46}$ C. Adamo and V. Barone, J. Chem. Phys. 110, 6158 (1999).

${ }^{47}$ Y. Zhao and D. Truhlar, Theor. Chem. Acc. 1, 215 (2008).

${ }^{48}$ J. Hepburn, G. Scoles, and R. Penco, Chem. Phys. Lett. 36, 451 (1975).

${ }^{49}$ R. Ahlrichs, R. Penco, and G. Scoles, Chemical Physics 19, 119 (1977).

${ }^{50}$ S. Grimme, J. Comp. Chem. 27, 1787 (2006).

${ }^{51}$ E. Ruiz, D. R. Salahub, and A. Vela, J. Phys. Chem. 100, 12265 (1996).

${ }^{52}$ J. Perdew, R. Parr, M. Levy, and J. Balduz Jr, Phys. Rev. Lett. 49, 1691 (1982).

${ }^{53}$ A. Cohen, P. Mori-Sánchez, and W. Yang, Phys. Rev. B 77, 115123 (2008).

${ }^{54}$ A. Cohen, P. Mori-Sánchez, and W. Yang, Science 321, 792 (2008).

${ }^{55}$ M. Valiev, E. Bylaska, N. Govind, K. Kowalski, T. Straatsma, H. V. Dam, D. Wang, J. Nieplocha, E. Apra, T. Windus, and W. de Jong, Comp. Phys. Comm. 181, 1477 (2010). 